\title{
Evaluación de la constancia de la energía empleando un sistema de imagen portal
}

\section{Using an Electronic Portal Imaging Device for routine energy constancy assessment}

\section{Leticia Irazola Rosales, Álvaro Perales Molina, Borja Aguilar Redondo, Luis Ramos García, Benigno Barbés Fernández}

Servicio de Radiofísica y Protección Radiológica, Clínica Universidad de Navarra.

Fecha de Recepción: 07/11/2019 - Fecha de Aceptación: 15/01/2020

Objetivo: Presentar una metodología para el control diario de la calidad energética del haz en rutina clínica, alternativa al uso de sistemas comerciales, empleando el sistema de imagen portal en combinación con un par de piezas cilíndricas de aluminio.

Método: Degradando externamente el haz de fotones, se consiguió una desviación en el parámetro representativo de la energía (PDD20,10: cociente de porcentaje de dosis a las profundidades de 20 y $10 \mathrm{~cm}$, respectivamente) de en torno al $2 \%$, considerada como clínicamente relevante. La metodología propuesta se validó para la detección de esta perturbación del haz.

Resultados: Se observó que, de manera reproducible, el método era capaz de distinguir las desviaciones efectuadas en la energía del haz, y se establecieron unos valores umbral de alarma.

Conclusiones: La metodología propuesta permite detectar desviaciones clínicamente relevantes de la energía, mediante el uso del sistema de imagen portal y dos piezas cilíndricas de aluminio. El sistema ha demostrado ser fiable y sencillo de implementar, sin requerir equipamiento adicional.

Palabras clave: Control de calidad diario, imagen portal, factor de calidad del haz.

Introduction: In the present work, we aimed to present an alternative methodology to the use of commercial devices for daily quality assurance of the linac energy in the clinic using the electronic portal-imaging device combined with a pair of cylindrical aluminum pieces.

Methodology: With an external degradation of beam energy, we achieved a modification of around $2 \%$ (considered as clinically relevant) in the representative energy parameter ( $P D_{20,10}$ : ratio of dose percentage at 20 and $10 \mathrm{~cm}$ depths, respectively). The proposed methodology was then validated for the detection of this beam perturbation.

Results: We observed that the methodology was able to distinguish the intentioned deviations performed in photon energy with a good reproducibility, obtaining threshold-warning levels.

Conclusions: The new methodology allows to establish a warning in case of a deviation clinically relevant in beam energy quality with the only use of the portal-imaging system together with a couple of aluminium pieces. The system has demonstrated to be reliable as well as easy to implement without needing extra equipment.

Key words: Daily quality control, EPID, beam quality factor.

\section{Introducción}

Habitualmente, en el ámbito de la física médica, se emplea el término calidad del haz de un acelerador lineal como análogo a la energía del mismo. Aunque en la literatura existen diversos parámetros descripti- vos de la calidad del haz, los más empleados suelen ser el porcentaje de dosis absorbida en profundidad (Percentage Dose Depth, PDD) y la razón tejido maniquí (Tissue-Phantom Ratio, TPR), basados en la penetración de un haz en un medio homogéneo como es el agua. ${ }^{1}$ El protocolo español, basándose en las

*Correspondencia: lirazola@unav.es

https://doi.org/10.37004/sefm/2020.21.1.003 
indicaciones de la IAEA (International Atomic Energy Agency) y AAPM (American Association of Physics in Medicine), establece distintas periodicidades para evaluar la constancia de este parámetro, según el sistema de verificación empleado, a saber: diario para un sistema de verificación rápida, mensual para una verificación con maniquí sólido y anual para el caso de una determinación directa del TPR 20,10 . 2,3 En el documento TG-40 de la AAPM se establece una evaluación de la constancia del factor de salida del campo (dosis por unidad monitor, medida en condiciones estándar) con una periodicidad mensual. ${ }^{3-5}$

Tradicionalmente, en las medidas de control de calidad diario, se emplean sistemas de verificación rápida con equipos comerciales basados en matrices de detectores, tales como QuickCheck ${ }^{\text {webline }}$ (PTW, Freiburg, Germany), StarTrack (IBA Dosimetry, Schwarzenbruck, Germany) o Daily $Q A^{T M} 3$ (Sun Nuclear Corporation, Melbourne, FL). Estos equipos presentan una fiabilidad aceptable dada la alta reproducibilidad y adecuada incertidumbre en la medida. ${ }^{1}$ En este tipo de dispositivos, se evalúa un parámetro representativo de la calidad del haz (Beam Quality Factor, BQF) y se estudian las posibles desviaciones diarias respecto a un valor considerado como referencia. Estos sistemas suelen emplear un filtro metálico (usualmente de aluminio o plomo) para atenuar la lectura obtenida respecto al valor central, siendo el cociente entre estos dos valores considerado como subrogado de la calidad del haz. El análisis estadístico del parámetro analizado por el dispositivo, a través del cálculo de su varianza, permite conocer la constancia de la calidad del haz estudiado.

En el caso del equipo QuickCheck, el BQF para fotones se determina a partir de la lectura de una de las cámaras ventiladas (no central) disponibles para fotones ( $E_{i}$, donde $i$ representa la energía a estudio, disponiendo de distintos espesores de material absorbente sobre el detector) y la del eje central (Central Axis, CAX) para un campo $10 \times 10 \mathrm{~cm}^{2}$. Entonces, a partir de una relación polinómica, proporciona un valor como subrogado de la calidad del haz (BQF), con una precisión de cuatro decimales (los detalles de este ajuste no se encuentran disponibles para los usuarios ${ }^{5}$ ). Para este detector, empleado en condiciones de referencia, los estudios apuntan a un comportamiento aproximadamente lineal entre la variación de la energía y el parámetro BQF. Así, la recomendación en los valores límite de desviación de este parámetro suelen estar en torno al $5 \%$, sin superar el $\pm 10 \%$. $^{5-7}$

Por otro lado, el sistema StarTrack presenta unas placas adicionales para la evaluación de la constancia de la energía, empleando un algoritmo a partir del cociente de la señal de las cámaras. Finalmente, en el equipo Daily QA-3 la energía de fotones se verifica evaluando las variaciones en la planitud (flatness) del haz en medidas consecutivas, empleando las cuatro cáma- ras de ionización (a $11.3 \mathrm{~cm}$ del centro) y la cámara central, considerando como valores límite desviaciones del $3 \%{ }^{8}$

Los principales inconvenientes de estos dispositivos son el tiempo empleado en su posicionamiento, proceso que además puede introducir variaciones en la homogeneidad y simetría del haz, junto con las incertidumbres asociadas al propio sistema de medida. Diversas publicaciones sugieren el uso de un sistema de imagen portal (Electronic Portal Imaging Device, EPID) como método alternativo para el control de calidad diario a través de un análisis basado en la imagen. ${ }^{9-12}$ Una vez evaluado el sistema y controlado su funcionamiento (siguiendo las guías de la AAPM), diversos estudios han demostrado su uso como alternativa para la realización de controles de calidad inherentes al haz de radiación. ${ }^{13-14}$ Muchas de las pruebas establecidas en los programas de garantía de calidad de los aceleradores lineales se llevan a cabo en diversos centros de manera rutinaria empleando sistemas de imagen portal como la verificación de los tamaños de campo, la posición de las láminas de los colimadores multilámina (MLC), la coincidencia de isocentros de $\mathrm{kV}$ y MV, y la dosimetría relativa: planitud, simetría, calidad del haz y congruencia campo de luz-radiación. Cabe resaltar la importancia de la caracterización del EPID, en términos de periodicidad y tolerancia para su uso en control de calidad diario.

En nuestro centro, el control de calidad diario para la evaluación de los parámetros citados se lleva a cabo desde hace 4 años con el sistema de imagen portal, a partir de la irradiación de campos abiertos, con una duración aproximada de unos cinco minutos. Se toman siete imágenes: una de tamaño de campo $10 \times 10 \mathrm{~cm}^{2}$ para cada energía, una de $20 \times 20 \mathrm{~cm}^{2}$ y otra con el colimador girado $180^{\circ}$. Las imágenes son analizadas en un programa de Matlab, para evaluar la constancia del campo de radiación en términos de simetría, planitud, tamaño y posicionamiento del mismo, así como el factor de salida. Este último parámetro se considera a partir de la dosis depositada en el panel, empleando como subrogado el ennegrecimiento de la imagen. Para obtener una evaluación completa del haz siguiendo esta metodología, se requiere evaluar también la constancia de la calidad del haz. Por ello, empleando las medidas realizadas en el control de calidad diario, este trabajo propone un método basado en la atenuación del haz al atravesar dos cilindros de aluminio situados en posiciones fijas sobre la superficie del EPID, dentro del haz de radiación, pero periféricas para no perturbar el resto de parámetros analizados en el control calidad diario. De esta forma, aprovechando la tanda de medidas realizadas para el estudio de parámetros geométricos del haz, se pretende evaluar un parámetro representativo de calidad del haz (análogo al BQF), de manera adicional y sin requerir medidas extras en el procedimiento. Para evaluar la bondad de la metodología propuesta, se 
realizaron las mismas medidas empleando dos dispositivos de PTW (QC-6 y QuickCheck) y se compararon los resultados. Cabe destacar que el propósito de este sistema no es dar un valor exacto de la desviación de la energía, sino proporcionar un indicador sensible a una desviación reseñable en la energía del haz, señalando la necesidad de realizar medidas más exhaustivas.

\section{Material y métodos}

Las mediciones de este trabajo se llevaron a cabo en dos aceleradores lineales Elekta Versa HD (Elekta AB, Stockholm Sweden) con el sistema iViewGT para la imagen portal. Este EPID consiste en un panel detector de Si amorfo de $41 \times 41 \mathrm{~cm}^{2}\left(26 \times 26 \mathrm{~cm}^{2}\right.$ en isocentro), que permite la obtención de imágenes de $1024 \times 1024$ píxeles de 16 bits. El procesado de imágenes fue llevado a cabo empleando Matlab2019a (R9.6, TheMathWorks Inc., Natick, MA, 2000).

\subsection{Caracterización del sistema de imagen portal}

\subsubsection{Evaluación de la constancia}

Para asegurar que la metodología propuesta proporciona un margen de confianza adecuado para detectar posibles desviaciones en la energía del haz, se estudió la constancia del factor de salida (evaluado rutinariamente en términos de valor de pixel en el centro del panel, considerando los $101 \times 101$ pixel centrales de este) durante un periodo de 4 meses. Se emplearon las mismas condiciones de irradiación del control de calidad diario: $100 \mathrm{UM}$ en un campo $10 \times 10 \mathrm{~cm}^{2}$ para todas las energías disponibles en la máquina ( 6 y $10 \mathrm{MV}$ en modalidad Flattening Filter Free (FFF), y 6 , 10 y 15 MV con el filtro aplanador).

\subsubsection{Evaluación de la linealidad}

Se analizó también la respuesta de ambos sistemas de imagen portal en el rango 95 a 105 UM, en intervalos de 1 UM. El estudio se realizó con y sin cilindros, para evaluar la posible modificación en la respuesta al interponer estos al haz. ${ }^{15}$

\subsection{Perturbación de la energía del haz}

Para evaluar la sensibilidad del método propuesto para el estudio de la constancia de la energía del haz, se propuso degradar la misma. Puesto que su modifi- cación a partir del ajuste de parámetros del acelerador como la corriente del imán de deflexión (Bending Magnet Current, BMI) supone la intervención del servicio de mantenimiento y compromete el tratamiento de pacientes mientras se realizan las medidas, se consideró más adecuada una modificación externa del mismo. Desviaciones en el BMI de un $\pm 10 \%$ para haces FF y FFF, implican un cambio de alrededor de $\pm 1.5 \%$ en la medida de rendimiento en profundidad (PDD). ${ }^{6,16}$ Considerando estos valores como desviaciones clínicamente relevantes, se procedió a degradar la energía en porcentajes similares.

El sistema elegido para modificar el haz consistió en la colocación de unas láminas metálicas a la salida de este (Fig. 1) que redujera la contribución de las energías bajas. Con el objeto de conseguir la degradación deseada, se realizaron diferentes pruebas en términos de material, distancia y número de láminas para cada energía, optando finalmente por un sistema de láminas de cobre de $10 \times 10 \mathrm{~cm}^{2}$ y $1 \mathrm{~cm}$ de espesor, ubicadas aproximadamente a $10 \mathrm{~cm}$ de la retícula del haz (Fig. 1). Las desviaciones conseguidas fueron evaluadas con un diodo IBA PFD3G 322-600 en una cuba de agua, según el protocolo establecido. ${ }^{4}$ Para mayor seguridad, las medidas se realizaron dos veces, con un mes de separación entre ellas, obteniendo resultados comparables.

\subsection{Análisis con cilindros}

\subsubsection{Sistema de medida}

Una vez conseguida la degradación deseada en la energía, para garantizar la sensibilidad y reproducibilidad del sistema, se evaluaron distintos espesores de cilindros (de $3.75 \mathrm{~cm}$ de diámetro) y posicionamiento de estos sobre el sistema de imagen. Como el objetivo era implementar este procedimiento en el control de calidad rutinario en la clínica, se estudió la posible influencia del uso de los cilindros en el análisis de los restantes parámetros de interés: factor de salida, simetría, planitud de haces convencionales o perfil de haces FFF, centrado del haz y tamaño de campo. Se compararon diariamente los valores obtenidos con y sin los cilindros durante un periodo superior a un mes.

Para la elección del sistema de cilindros más adecuado, se hicieron diversas pruebas colocando primero cuatro cilindros de espesores comprendidos entre $0.5 \mathrm{y}$ $3 \mathrm{~cm}$, evaluando la variación en el parámetro de factor de corrección de energía (FCE) obtenido para cada energía (inicialmente en los cuatro cuadrantes centrales del campo). Una vez encontrado la disposición óptima (Fig. 2), se evaluó la desviación que implicaba esta 

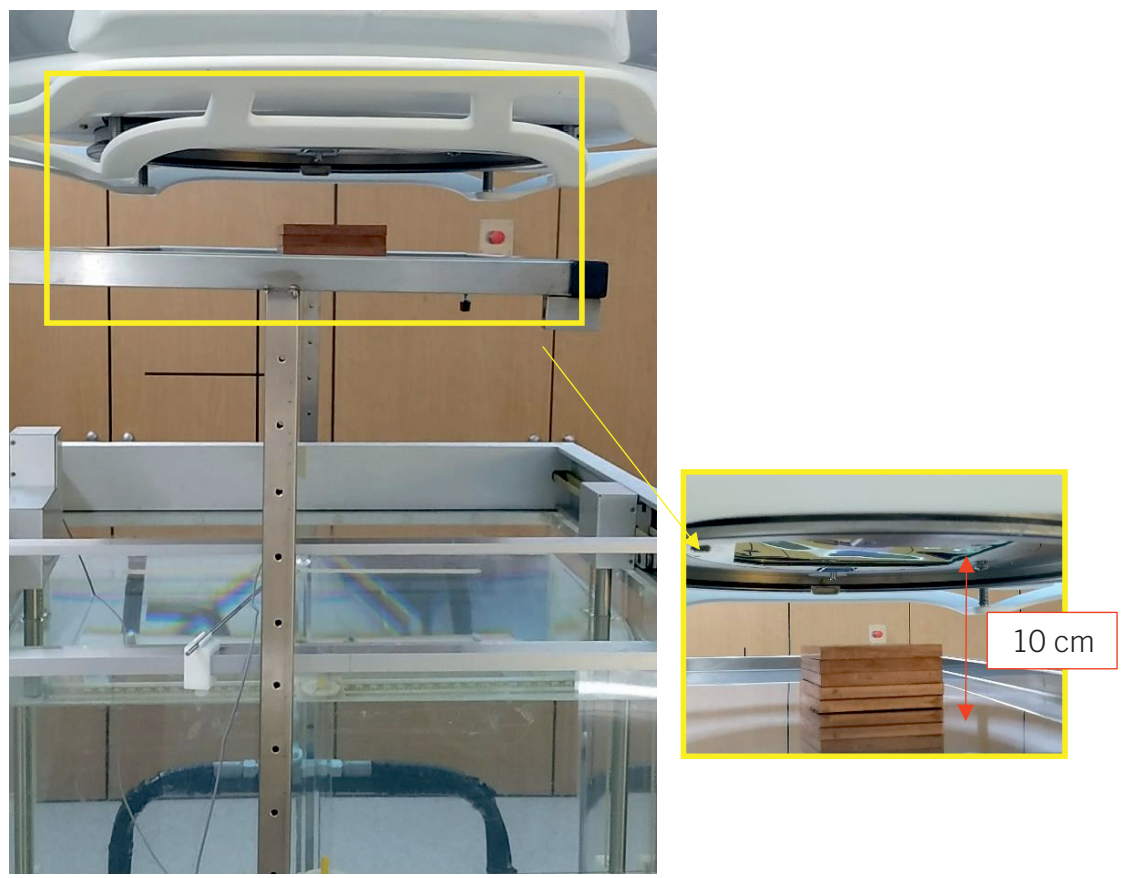

Fig. 1. Sistema basado en el uso de láminas de Cu de $10 \times 10 \mathrm{~cm}^{2}$ y $1 \mathrm{~cm}$ de grosor, empleadas para modificar el haz de fotones.

distribución, analizando tanto los perfiles y como los resultados del proceso de control de calidad durante días sucesivos con y sin el sistema de cilindros. El espesor de los mismos, se fijó tras analizar la constancia de los valores FCE, ${ }^{1}$ de acuerdo con los más reproducibles para el conjunto de energías a analizar.
Se repitieron al menos diez veces todas las medidas, en distintos días, para asegurar su reproducibilidad. Estas medidas fueron realizadas también en otro acelerador Versa HD (con un modelo de EPID posterior), empleando una geometría de medida análoga, con resultados equivalentes.

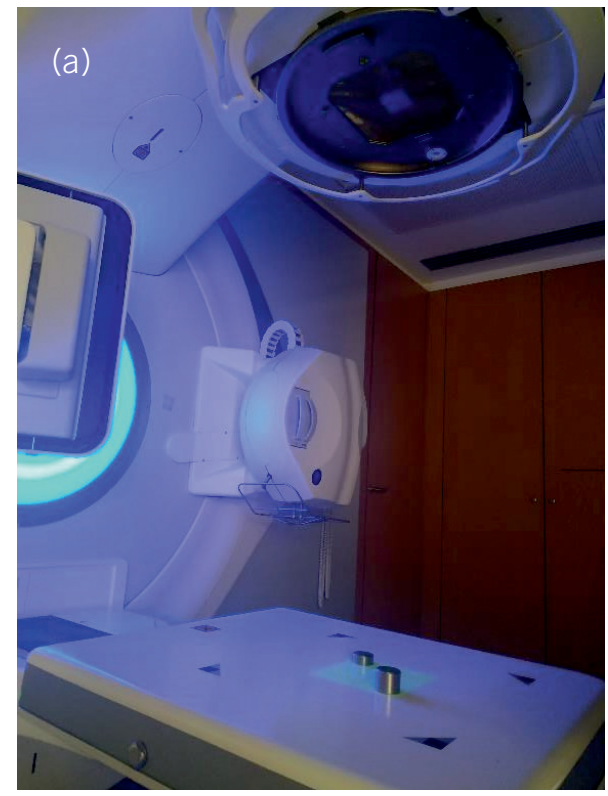

(b)

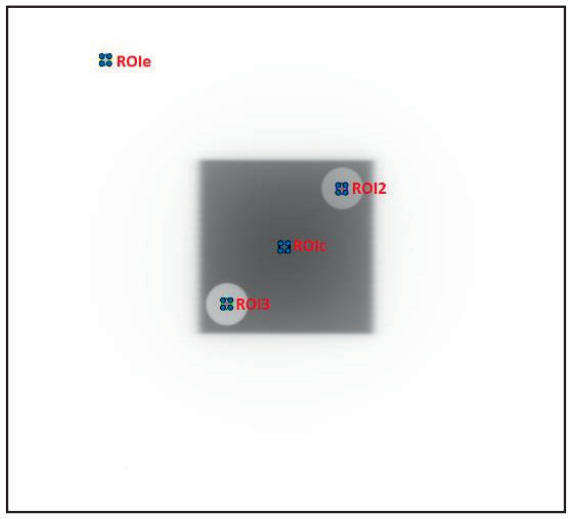

Fig. 2. (a) Montaje de medida con sistema de 2 cilindros y (b) imagen obtenida con el EPID tras ser analizada con Matlab. 


\subsubsection{Análisis de imágenes}

Una vez adquiridas las imágenes para las distintas energías en condiciones de referencia $\left(10 \times 10 \mathrm{~cm}^{2}\right.$, 100 UM) con el EPID, fueron exportadas en formato TIFF (Tagged Image File Format) junto con un fichero ASCII (American Standard Code for Information Interchange) que contiene un factor de normalización en valor de pixel que es aplicado automáticamente por el programa de adquisición de imágenes portal. Se procesan las imágenes para localizar el centro de los cilindros y poder tomar una región de análisis (Region Of Interest, ROI). En primer lugar, se realiza una discretización binaria de las mismas, empleando diferentes sensibilidades hasta lograr la detección de los círculos correspondientes a los cilindros colocados durante la medida. Una vez localizados, se toman ROls circulares de la cuarta parte del radio del círculo detectado, y se calcula el valor promedio de píxel en estas ROI, para obtener el factor de calidad de energía $(F C E)$ :

$$
F C E=\frac{\left(R O I_{i}-R O I_{e}\right)}{\left(R O I_{c}-R O I_{e}\right)}
$$

donde (ver Fig. 2b):

- $R O I_{i}$ : en el círculo correspondiente al cilindro $i$ a estudiar

- $R O I_{e}$ : en un punto fuera del campo de irradiación

- $R O I_{c}$ : en el centro del campo, fuera de los círculos

Como este sistema pretende ser un indicador sensible a una desviación del orden del $2 \%$ en el $\mathrm{PDD}_{20,10}$, se evaluó la constancia tanto de los valores de cada $\mathrm{ROI}$, como de los parámetros FCE para cada una de las cinco energías disponibles, en ambos aceleradores lineales.

\subsection{Comparación con otros sistemas}

Para comparar la capacidad de detección de constancia en la energía de la metodología aquí presentada, se interpusieron a un haz de $15 \mathrm{MV}$ distintos grosores de $\mathrm{Cu}$ (con el objeto de generar distintas modificaciones en la energía), y se estudió el cambio del haz por distintos métodos: el propuesto en este trabajo, la medida de PDD con cuba de agua (siguiendo el protocolo TG-40), un equipo QuickCheck y un equipo QC-6. En caso de QuickCheck, se analizó el parámetro obtenido directamente con el programa comercial (BQF); en el caso del QC-6, se comparó el cociente entre la cámara central y una de las ubicadas en las esquinas del campo.

\section{Resultados}

\subsection{Caracterización del sistema de imagen portal}

Los resultados obtenidos en la desviación estadística del factor de salida medido en el control de calidad diario con el sistema de imagen portal en el período de duración de este estudio, mostraron desviaciones menores del $1 \%$.

En el caso del análisis de la respuesta del sistema portal al irradiar con diferente número de UM, se obtuvieron valores de $\mathrm{R}^{2}$ superiores a 0.99 para el ajuste lineal, en todas las energías disponibles para ambos aceleradores. Se verificó también de manera adicional que el montaje de cilindros no perturbaba dicha linealidad. Eventualmente, se comprobó que una nueva calibración del EPID no afectaba a la linealidad de la respuesta ni al FCE (conviene tener en cuenta que la respuesta no procesada de ese panel no es lineal, ni lo es el proceso de calibración).

\subsection{Degradación de la energía del haz}

Se comprobó que el sistema empleado para la degradación del haz (ver 2.2) no modifica sustancialmente parámetros característicos del haz, como la planitud y simetría de los perfiles o la forma de los PDD (Fig. 3). Fue necesario aumentar la tasa de muestreo de las medidas con diodo para disminuir el ruido, dada la disminución de fluencia causada por la interposición de las láminas de $\mathrm{Cu}$.

Los resultados de las variaciones del parámetro $\mathrm{PDD}_{20,10}$ con y sin el montaje empleado para el degradado obtenidos se muestran en la Tabla 1. Tal y como se ha mencionado previamente, esta perturbación del haz pretende hacerlo más penetrante, generando una modificación en la energía que podría tener repercusión clínica. ${ }^{15}$

Se verificó adicionalmente que el panel plano mide de forma fiable a fluencias bajas, de forma que la disminución de la fluencia causada por la degradación del haz era reproducible.

\subsection{Análisis con cilindros}

Con una configuración inicial en que se situaban 4 cilindros en los cuadrantes centrales, se observó una alteración importante tanto en los perfiles como en los 
Crossplane
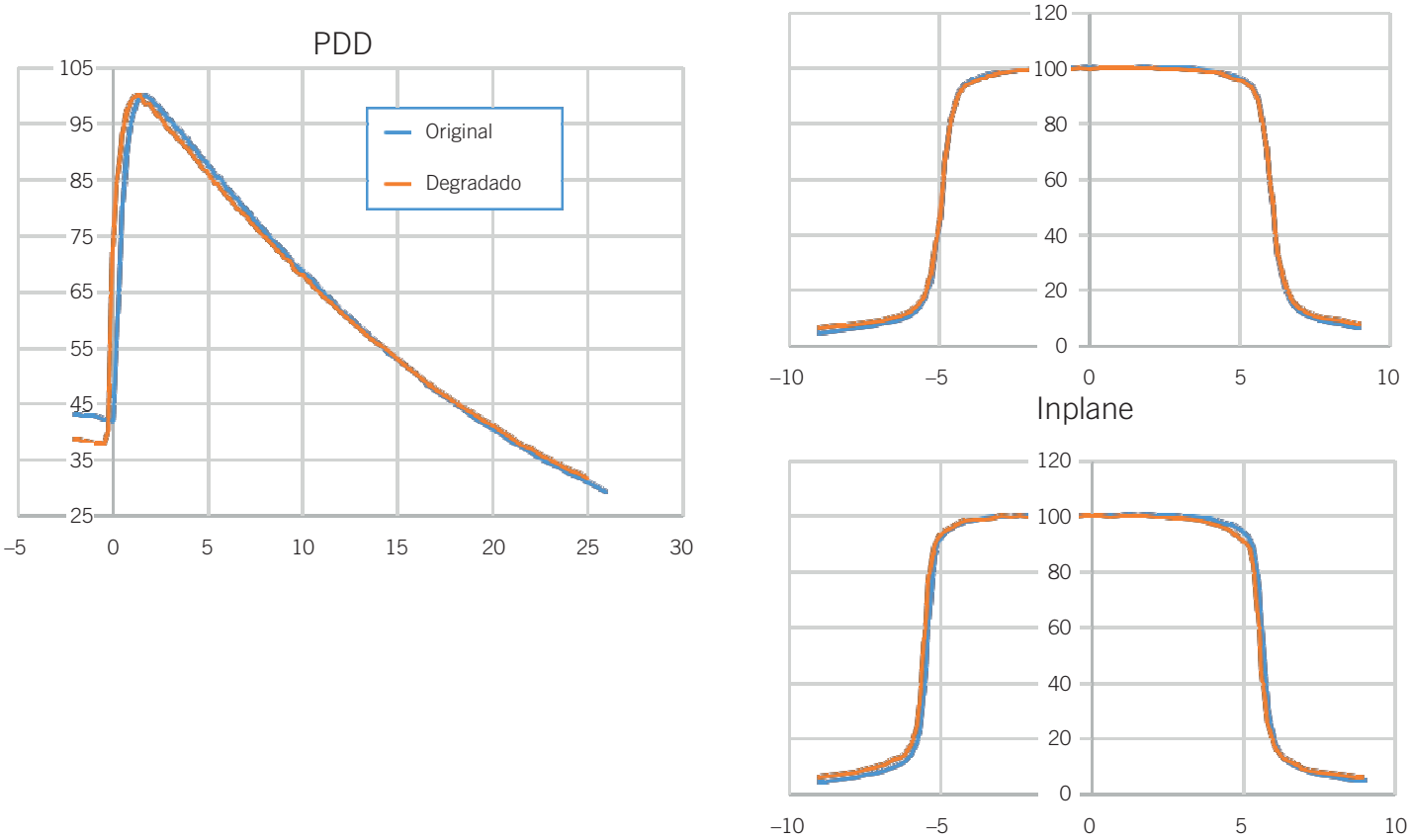

Fig. 3. Representación gráfica de los parámetros del haz al degradarlo con el sistema de bloques de Cu.

parámetros indicativos de la calidad del haz: factor de salida, tamaño de campo, forma de los perfiles, simetría. Para evitarlo, se modificó el sistema de medida alejando los cilindros del centro. En vista de los valores obtenidos y considerando su reproducibilidad, se consideró más adecuado utilizar el cilindro de menor espesor $(1.5 \mathrm{~cm}$, denotado por FCE2) para las energías sin filtro de 6 y $10 \mathrm{MV}$, y el cilindro de mayor espesor ( $3 \mathrm{~cm}$, FCE3) para las energías con filtro de 6, 10 y $15 \mathrm{MV}$, colocados en las posiciones que se muestran en la Fig. 2.

La desviación típica encontrada para esta última configuración entre ambos montajes (con el sistema elegido y sin cilindros) se encuentra por debajo del $1 \%$

Tabla 1. Desviaciones obtenidas en el parámetro $\mathrm{PDD}_{20,10}$ y modificación en el output factor (indicador de la fluencia) para todas las energías disponibles, con el sistema de láminas de $\mathrm{Cu}$.

\begin{tabular}{c|c|c|c} 
Energía & $\begin{array}{c}\text { No láminas/ } \\
\text { espesor Cu (cm) }\end{array}$ & $\begin{array}{c}\text { Desv(\%) } \\
\text { PDD }_{20,10}\end{array}$ & $\begin{array}{c}\text { Desv(\%) } \\
\text { f. salida }\end{array}$ \\
\hline $6 \mathrm{MV} \mathrm{FFF}$ & 1 & $2.0 \pm 0.6$ & $41.6 \pm 0.2$ \\
\hline $6 \mathrm{MV}$ & 2 & $2.1 \pm 0.3$ & $58.5 \pm 0.1$ \\
\hline $10 \mathrm{MV}$ FFF & 2 & $2.3 \pm 0.0$ & $57.2 \pm 0.2$ \\
\hline $10 \mathrm{MV}$ & 5 & $2.2 \pm 0.3$ & $81.9 \pm 0.1$ \\
\hline $15 \mathrm{MV}$ & 7 & $2.1 \pm 0.3$ & $89.5 \pm 0.1$
\end{tabular}

para todos los parámetros estudiados en el control de calidad diario, compatible con la variación diaria medida para el propio sistema de imagen portal.

Los resultados obtenidos para la constancia y la desviación porcentual del parámetro FCE al degradar el haz sirvieron para establecer los valores límite a modo de alarma, indicativa de una variación significativa en la calidad de energía esperada (aproximadamente $2 \%$ ), mostrados en la Tabla 2, representando la variación porcentual respecto al caso de la energía sin degradar.

\subsection{Comparación con otros sistemas}

Los resultados de las desviaciones porcentuales respecto al valor diario del parámetro $B Q F$, obtenidos al medir con QuickCheck (empleado habitualmente en otro acelerador Versa), se muestran a modo de comparación en la última columna de la Tabla 2.

En la Fig. 4, se muestran las desviaciones porcentuales obtenidas con respecto a la medida sin degradar en dos de los métodos estudiados para la evaluación de la degradación en 15 MV: obtención del parámetro $\mathrm{PDD}_{20,10}$ en cuba de agua, y el parámetro FCE propuesto en este trabajo. Se observa un comportamiento aproximadamente lineal con el aumento de láminas interpuestas en el haz para conseguir la degradación deseada. Para energías menores, la linealidad es más acusada. En base a estos resultados, el 
Tabla 2. Valores promedio y desviaciones porcentuales consideradas como "alarma" (indicando una variación importante en la calidad de la energía del haz) en el valor del parámetro FCE (Factor de Calidad de Energía) en las dos cilindros empleadas (2 y 3) al degradar la energía con el sistema propuesto. En la última columna se muestran las desviaciones encontradas con el QC de PTW entre el haz normal y el modificado con el sistema de Cu.

\begin{tabular}{c|c|c|c|c|c} 
Energía & FCE $2\left(\times 10^{-1}\right)$ & Alarma FCE 2 (\%) & FCE 3 & Alarma FCE 3 (\%) & $\Delta$ BQF \\
\hline 6 MV FFF & $5.491 \pm 0.004$ & 4.5 & $3.716 \pm 0.005$ & 7.0 & 20.6 \\
\hline 6 MV & $6.599 \pm 0.003$ & 2.5 & $4.467 \pm 0.004$ & 4.0 & 17.8 \\
\hline $10 \mathrm{MV} \mathrm{FFF}$ & $5.693 \pm 0.006$ & 6.0 & $3.964 \pm 0.004$ & 8.5 & 17.8 \\
\hline $10 \mathrm{MV}$ & $7.461 \pm 0.004$ & 1.5 & $5.290 \pm 0.004$ & 2.0 & 22.3 \\
\hline $15 \mathrm{MV}$ & $7.856 \pm 0.003$ & 2.0 & $5.627 \pm 0.003$ & 2.5 & 18.4
\end{tabular}

FCE puede servir como criterio de "alarma" fiable para desviaciones del orden del $2 \%$ en la energía, sin ser un método de medida de dicha energía.

Al medir el haz degradado con los dispositivos comerciales, observamos comportamientos inesperados, quizá debidos al método de degradado. El parámetro $\mathrm{BQF}$, indicador de la calidad de energía del QuickCheck, se modifica en torno a un $20 \%$ y se mantiene más o menos constante para cualquier espesor de láminas empleado. En el caso del QC-6, se obtiene un comportamiento más próximo al esperado, con desviaciones comprendidas entre un 0.1 y $1.5 \%$ para el parámetro representativo de la calidad energética del haz.

\section{Discusión}

Las bajas desviaciones obtenidas en el uso del EPID para el control de calidad diario, avalan la fiabilidad del sistema para su uso en rutina clínica. Además,

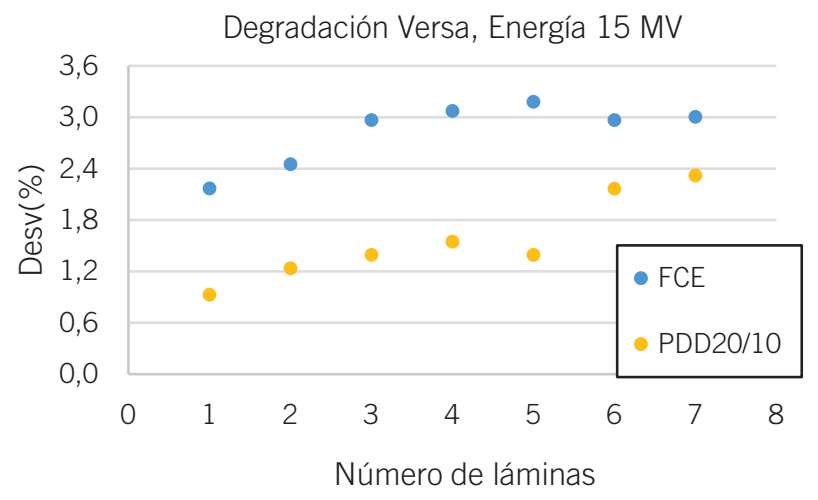

Fig. 4. Representación gráfica de las desviaciones porcentuales obtenidas en los parámetros PDD $_{20,10}$ (analizado con cuba) y el FCE (Factor de Calidad de Energía) cuando se realiza una degradación empleando distinto número de láminas de Cu en la energía de 15 MV y se compara con la energía normal. los valores obtenidos se hallan en concordancia con los estimados por otros grupos para este mismo tipo de EPID, ${ }^{15}$ al igual que sucede para el estudio de la linealidad. Los valores medidos nos permiten considerar que las desviaciones obtenidas con el sistema y las tolerancias indicadas en este manuscrito se pueden tomar como un indicador adecuado para una posible variación en la energía, despreciando las posibles fluctuaciones debidas a las características intrínsecas del sistema de imagen.

El método propuesto para la degradación de la energía del haz, consistente en interponer unas láminas de Cu entre este y el EPID sin tener que modificar los ajustes del haz en el linac, resultó eficaz y reproducible. Las desviaciones obtenidas en el parámetro $\mathrm{PDD}_{20,10}$ nos permiten verificar que, efectivamente, se consigue modificar la calidad del haz en torno a un $2 \%$, valor que consideramos clínicamente relevante (como nivel de acción). Se observó una buena reproducibilidad de este sistema evaluada tanto con la medida de $\mathrm{PDD}_{20,10}$ como con la evaluación de la fluencia que alcanza el EPID (resultados mostrados en Tabla 1). La constancia de los valores FCE obtenidos junto con la reproducibilidad de la metodología empleada para la degradación del haz, hacen que el sistema de cilindros aquí propuesto resulte un sistema adecuado a modo de alarma para la posible variación en la calidad de la energía del acelerador clínico para usar en el control de calidad diario.

Las pequeñas desviaciones obtenidas en la estimación de los parámetros que se analizan de forma diaria en el acelerador clínico con el sistema de EPID (factor de salida, forma de los perfiles, simetría, tamaño de campo, etc.) al emplear el sistema de cilindros descrito, lo convierten en una metodología apta para su implementación en rutina clínica, y más completo que el uso de detectores dedicados. Además, la constancia de los valores del parámetro FCE, introducido aquí como un subrogado para la evaluación de la constancia de la calidad del haz, permite el establecimiento de unas alarmas propias de cada acelerador clínico y 
que resultan fiables como indicador de la necesidad de ajustar la energía analizada.

Los valores y la variabilidad observada del parámetro FCE (para ambos cilindros), nos permitió definir unos valores orientativos como referencia y alarma (desviaciones porcentuales de la referencia), respectivamente. Consideramos que, si se desea introducir esta metodología en otro centro o acelerador clínico, se debería realizar un análisis para evaluar tanto el espesor de cilindro necesario como los valores alarma para adaptarlos a las condiciones específicas, siguiendo el procedimiento que se ha expuesto en este estudio.

Finalmente, la comparación de los resultados obtenidos con las desviaciones indicadas por los sistemas comerciales QuickCheck y QC-6, y el uso de dos versiones distintas de panel plano del mismo fabricante, respaldan la metodología propuesta para su uso en los controles de calidad diarios en la clínica.

\section{Conclusiones}

Este trabajo presenta una metodología para la evaluación diaria de la constancia de la energía del haz empleando el propio sistema de imagen portal de los aceleradores clínicos. El montaje, basado en el uso de dos cilindros de aluminio directamente colocados sobre el EPID, se ha mostrado como un método reproducible para la detección de desviaciones en el parámetro representativo de la energía $\mathrm{PDD}_{20,10}$ de un $2 \%$, variación considerada relevante en la clínica. La realización repetida de este proceso en dos aceleradores clínicos, junto con su comparación con sistemas comerciales para el control de calidad diario, nos han permitido establecer unos valores alarma para su uso en rutina clínica, permitiendo por tanto detectar una posible desviación en la energía del haz y poder actuar en consecuencia.

\section{Bibliografía}

1. Pinza c, Lliso F. Control de Calidad en Aceleradores de Electrones para uso médico. Madrid SEFM;2009.

2. Adreo P, Burns DT, Hohlfeld K, Huq MS, Kanai T, Laiano F, Smyth $V$ and Vynkier S. IAEA TRS-398 Absorbed
Dose Determination in External Beam Radiotherapy: An International Code of Practice for Dosimetry based on Standards of Absorbed Dose to Water. IAEA 2006.

3. Klein EE, Hanley J, Bayouth J et al., Task Group 142 report: Quality assurance of medical accelerators. Med Phys 2009;36(9):4197-212.

4. KutcherGJ, Coia L, Gillin $M$ et al., Task Group 40: Comprehensive QA for Radiation Oncology. AAPM Report $n^{\circ}$ 46, April 1994.

5. Thwaites DI, Mijnheer B, Mills JA. Quality assurance of external beam radiotherapy. Radiation oncology physics: a handbook for teachers and students. Vienna: International Atomic Energy Agency; 2005. 407-450.

6. Mosleh-Shirazi MA, Rahimi S, Karbasi S. Medium-term stability of the photon beam energy of an Elekta Compact TM linear accelerator based on daily measurements of beam quality. IrJ Med Phys;2016:12(4):230-5.

7. True Beam Stability: Results After 6 Months https://www. wienkav.at/kav/kfj/91033454/physik/tb/tb_beam_stability. htm

8. Binny D, Lancaster CM, Kairn T, Trapp JV, Crowe SB. Technical note: Monitoring daily QA3 constancy for routine quality assurance on linear accelerators.Phys Medica 2016;32(11):1479-87.

9. Patallo S and García F. Uso del Sistema imagen portal electrónica iViewGT para control de calidad del haz de radiación de un acelerador lineal de uso clínico. Rev Cub Fis 2010;27(2B):251-6.

10. BudgellGJ, Zhang R and Mackay RI. Daily monitoring of linear accelerator beam parameters using an amorphous silicon EPID. Phys Med Biol 2007;52:1721-33.

11.Ding A, Xing $L$ and Han B. Development of an accurate EPID-based output measurement and dosimetric verification tool for electron beam therapy. Med Phys 2015;42:4190-8.

12. Saleh Z, Tang X, Song Y et al., Can EPID based measurement replace traditional daily output QA on Megavoltage linac?. Med Phys 2016;43(6)P26.

13. Wang y, Heaton R, Norrlinger B and Islam M. Quality assurance of electron beams using a Varian electronic portal imaging device. Phys Med Biol 2013;58:5461-75.

14. Andersson P. Utilizing statistical process control analysis and EPID for routine QA of medical linear accelerators. MS Lund University 2011.

15. Cilla S, Fidanzio A, Greco F, Sabatino D, Russo A, Gargiulo $L$, Azario $L$ and Piermattei $A$. Correlation functions for ElektaaSiEPIDs used as transit dosimeter for open fields.J ApplClin Med Phys2010:12(1):218-33.

16. Gao S, Balter PA, Rose M and Simon WE. A comparison of methods for monitoring photon beam energy constancy.J ApplClinMedPhys 2016;17(6):242-53. 


\section{FRAINLAB}

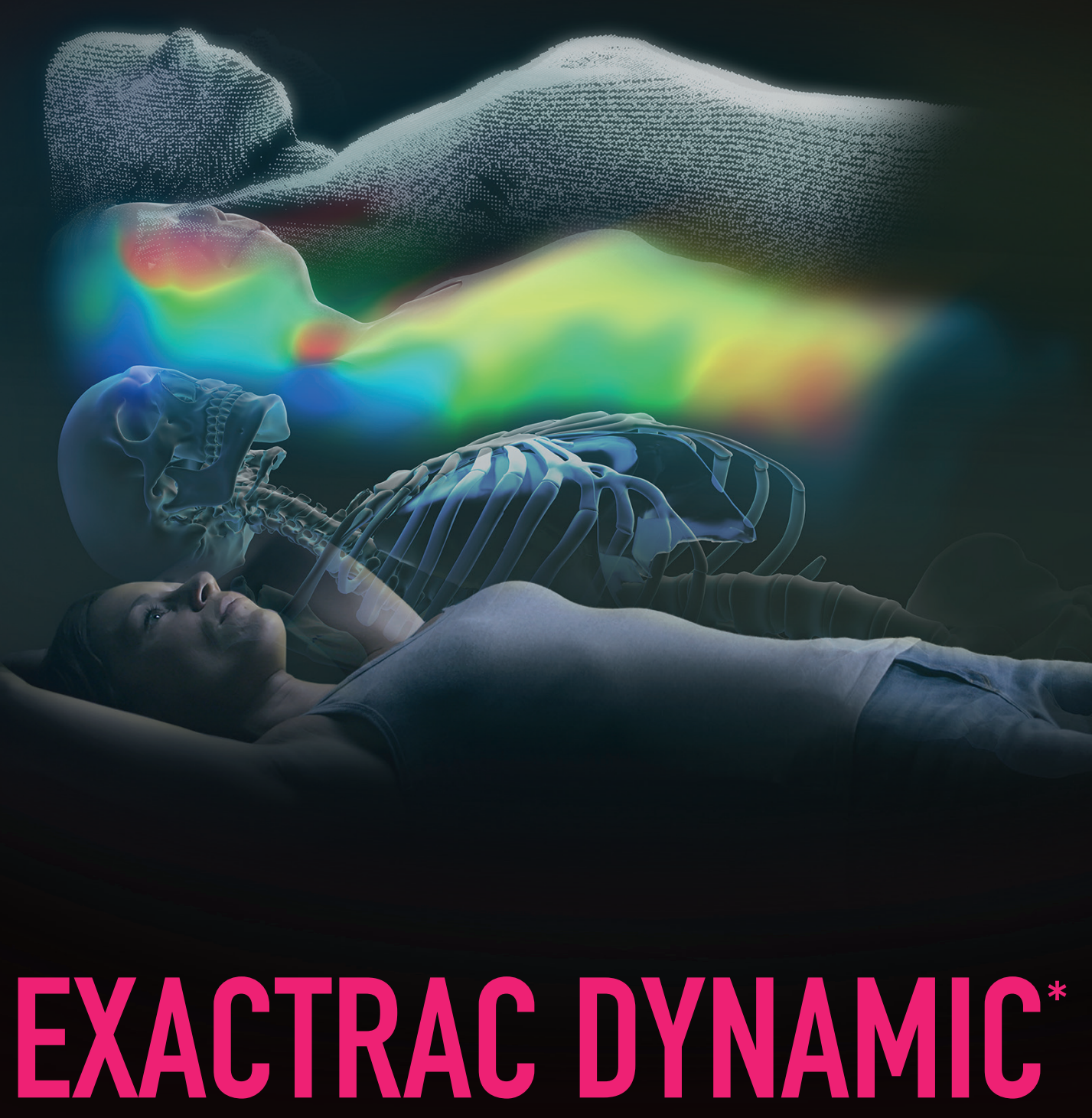

A New Dimension of Patient Positioning \& Monitoring

Learn how the next generation of ExacTrac is streamlining radiotherapy treatments for a broad range of clinical workflows at brainlab.com/exactrac. 